\title{
Set Based Concurrent Engineering Innovation Roadmap
}

\author{
Endris Temam Kerga ${ }^{1,2}$, Marco Taisch ${ }^{1}$, and Sergio Terzi ${ }^{3}$ \\ ${ }^{1}$ Politecnico di Milano, Piazza Leonardo da Vinci 32, 20133, Milan, Italy \\ endris.kerga@mail.polimi.it, marco.taisch@polimi.it \\ ${ }^{2}$ Carel Industries, Via della Industria 11, 35020, Brugine, Italy \\ endris.kerga@carel.com \\ ${ }^{3}$ Università degli studi di Bergamo, Marconi 5, 24044, Dalmine (BG), Italy \\ sergio.terzi@unibg.it
}

\begin{abstract}
SBCE (Set Based Concurrent Engineering) is an element of lean practice in product development (PD), and it composes theoretical principles to apply it at early phase of a design process. However, executing principles of SBCE in practice require extensive efforts. Thus, a systematic methodology is required to identify and priorities potential areas (product's subsystems, components, features) where SBCE brings its utmost benefits. This paper proposes such a methodology called SBCE Innovation Roadmap (SBCE IR) that is used as a guideline by product designers to begin SBCE processes. A case study on Adiabatic Humidification System (AHS) is discussed to elaborate the SBCE IR methodology. Furthermore, an experimental SBCE process has been conducted on rack subsystem that shows a significant cost reduction.
\end{abstract}

Keywords: Set Based Concurrent Engineering (SBCE), Theory of Inventive Problem Solving (TRIZ), Contradictions.

\section{Introduction}

The traditional approach to develop a product concept typically starts with breaking it into its subsystems, defining detail requirements for each module and deriving a small number of alternative solutions which appear to meet the initial requirements. Engineers then quickly assess the solutions and select one option to be pursued. This process however, rarely turns out to be linear in nature. Usually, engineers discover that the chosen design solution does not meet the requirements formulated at the onset and the choice may have resulted in a variety of adversities issues. A series of iterative loops follow to either modify the concept until it satisfies the requirements or start the process over by selecting a completely different design solution. Because of the iterative nature where engineers move from point to point in the realm of searching for feasible designs, this process has been termed Point Based Concurrent Engineering (PBCE) [1], [2].

SBCE is an alternative approach first used by Toyota [1]. It is started with dividing the product into small subsystems and modules, however, unlike in PBCE, no detailed requirements are defined for subsystems and engineers only identify broad targets for each module. Based on these targets, a much larger number of alternative solutions 
are developed early in the process. Then, designers test, analyze and build multiple solutions for every subsystem in parallel [3]. Unless designers have acquired sufficient knowledge through analysis, simulation, prototypes, and tests to eliminate an alternative, it will remain as a feasible design option. Finally, feasible design alternatives can be evaluated against objective criteria (cost, time, quality) for convergence.

Though the principles of SBCE are sound and the theoretical benefits are promising, there are fundamental impediments for its practical success. The following gaps are underlined in this paper.

- SBCE is an extensive process: - to conduct an SBCE process, designers should go through extensive phases such as exploring, communicating, testing, and converging sets. Doing all these requires considerable time, investment and capabilities. Thus, a systematic method is needed to identify and prioritize subsystems or components or design parameters a priori of pursuing such an extensive process. Otherwise, efforts made will be wasted without achieving value. This paper proposes such a methodology called SBCE IR that enables to breakdown design problems and derive rules for SBCE implementation.

- The use of tradeoffs in SBCE: - in extant literature, SBCE has strictly been related to use of trade-offs. Toyota, for example, reported to base on trade-off analyses for exploring, evaluating and communicating sets [2]. However, using trade-offs restricts the level of innovation. Altshuller, in his prominent theory of inventive problem solving (TRIZ), underscores the limitation of accepting trade-offs in design [4]. In TRIZ, trade-offs or compromises are not accepted, rather they are eliminated if innovative design solutions are sought to be discovered. Moreover, in practice, understanding design tradeoffs is often too complex. Thus, SBCE has to be discussed along with existing established theories of innovation both to simplify it and enhance innovation. If TRIZ is integrated with SBCE process, there will be significant methodological improvements in identifying potential areas for innovation. Moreover, the search efforts for new design solutions can be formalized. The proposed SBCE IR methodology integrates TRIZ and SBCE.

- 'Psychological inertia':- is another practical bottleneck for SBCE's success. It is a phenomenon in design practice, where designers often tend to explore solutions within known design spaces [5]. Thus, it often becomes unlikely for designers to observe 'out of the box' solutions. Although, the SBCE IR doesn't have a direct contribution to avoid the phenomena, it allows design problems to surface and TRIZ principles will help to explore innovative solutions.

In summary, the above gaps are important to address to make SBCE pragmatic. The contribution of this paper is to develop a systematic methodology that helps designers to identify and prioritize design problems for further SBCE implementation. In section 2, SBCE IR will be introduced. A case study on AHS is discussed in section 3. Section 4 is dedicated to briefly present the results obtained from an SBCE process on rack subsystem of AHS. Finally in section 5, conclusions of the paper and possible further researches needed are outlined. 


\section{SBCE IR}

SBCE IR can be described as a step wise methodology that guides product developers to make rational choices to pursue SBCE processes at a sub-system or component levels. It will be used for matured products, and when developers need to identify and prioritize improvement areas. Six steps are needed to build it for a product.

Before going in detail on the steps, the assumptions and criteria taken for its construction are three: (1) Identification of system contradictions lead to improvement areas: the SBCE IR bases on identifying and overcoming contradictions for a product. Identifying system contradictions will instigate potential areas where SBCE can be executed (i.e. exploration, communication, testing and convergence) [4,6]. There are two types of contradictions that need solutions, physical and technical. The former occurs when a product has a requirement that should exist in opposite states at the same time. The latter occurs when two different requirements cannot be achieved without one gets worse; (2) Improvement areas should be prioritized based on customer value information, it is not uncommon that designers spent considerable amount of their time developing features that the customers are not interested in [7, 8]. Thus, information on customer value is paramount to be integrated before starting on a particular SBCE process. Once contradictory requirements are identified, customers' judgment on importance can be used as one criterion to prioritize contradictions; (3) Improvement areas should be prioritized based on competitive advantages, while building the SBCE IR, competitor analyses are used as an additional criterion to prioritize contradictions (i.e. how important will solving a contradiction be for competitiveness?).

In sum, from the above discussions, it should be clear that SBCE IR is the identification, prioritization and mapping of contradictions (taking customer and competition as criteria). The steps (see also Figure 1) and the associated methods used to build SBCE IR are:

- Identify customer requirements and assign importance

- Assess competitors' products and set targets

- Identify system contradictions

- Identify dependent and independent contradictions

- Derive rules to prioritize and select contradictions, and

- Map contradictions to products' design factors ${ }^{1}$.

The first step is to gather the right customer requirements, and assign the relative importance vector $(0 \leq \mathrm{W} \leq 1)$ using a pairwise comparison method of AHP (Analytical Hierarchical Process) [9]. For each requirement, in step 2, the performance level of the design will then be evaluated against those of competitors' products. Then, by setting the present and target values, each requirement will be assigned the degree of difficulties, $\mathrm{D} \in\{0,1,2,3\}$, where $0,1,2,3$ represent that a requirement is already

${ }^{1}$ Design factors can be defined as design variables that designers have the opportunity to change in SBCE process (e.g. material types, design configurations, number of components to use in a design, etc.) 
achieved, easily achievable, moderately difficult to achieve, and difficult to achieve respectively [10]. Now each requirement has information related to customer importance (W) and competitive advantages (D). To set priorities $\mathrm{P}$ for requirements, an aggregation of $(\mathrm{P}=\mathrm{W} \mathrm{X} \mathrm{D})$ is used. If requirements are grouped based on categories (technical performance, cost, usability, etc.), they can be assigned ranks $(\mathrm{R}=1,2$, $3 . .$.$) within the respective categories according to their P$ values.

In the next steps 3 and 4, contradictory requirements will be identified and contradictions (T) can be defined. However, two contradictions can be either independent or dependent [11]. Two contradictions said independent if design solutions proposed to overcome one will not solve the contradiction in the other. Otherwise, they are dependent. Moreover, if solutions proposed to a contradiction potentially solve many other contradictions, then that will be considered as the root contradiction. In order to identify independent/dependent contradictions, a method called DEMATEL (Decision Making Trial and Evaluation Laboratory) is used [12]. It's aimed to find the direct/indirect relationships between variables (in this case contradictions), and use a matrix and causal diagram to express the casual relationships and influence level between variables in a complicated system.

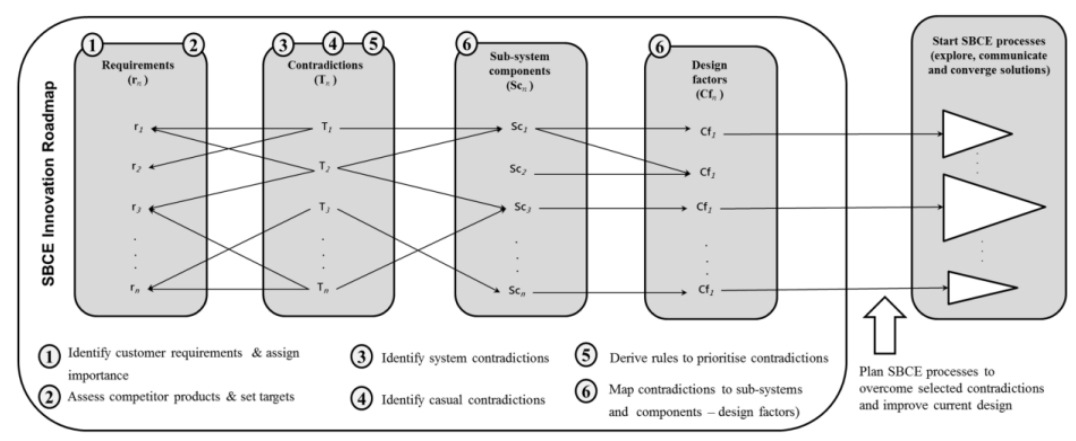

Fig. 1. Steps to build an SBCE IR for a product

Once the above steps are completed, rules to prioritize and select contradictions for further SBCE projects initiations will be the next step. In SBCE IR, three rules are identified: (a) rule of independent contradictions; select a dominating contradiction (b) rule of independent contradiction but tie; select based on experts' judgment; (c) rule of dependent contradictions, select the root contradiction. For example, assume three contradictions $\mathrm{T} 1, \mathrm{~T} 2, \mathrm{~T} 3$, with the ranks $(\mathrm{R})$ of the corresponding contradictory requirements as $\left(\mathrm{r}_{\text {up }}, \mathrm{r}_{\text {down }}\right) \sim(1,2),(1,4)$ and $(2,1)$ respectively. $T_{1}$ dominates $T_{2}$, but both $\mathrm{T}_{1} \& \mathrm{~T}_{2}$ are in tie with $\mathrm{T}_{3}$. Assuming all are independent, based on rule (a), designers should select $T_{1}$ over $T_{2}$ to initiate an SBCE project, but should impose judgment (other than customer importance and competitive advantage) to select either $\mathrm{T}_{1}$ over $T_{3}$ or $T_{2}$ over $T_{3}$ (rule b). However, if for example in step 4 , it is found that $T_{2}$ is the root contradiction for $\mathrm{T}_{1}$ and $\mathrm{T}_{3}$, then rule (c) is employed, and SBCE process should be initiated to search for sets of solutions to solve $T_{2}$, communicate and test the sets for convergence. 
The final step to build SBCE IR is to map selected contradictions to product's design factors. This can be done by experienced engineers that can associate the inherent design factors that have to be improved to solve the selected contradictions.

The steps provide logical approach to arrive to prioritized areas where SBCE process should be initiated. Furthermore, it enables designers to think about solutions for smaller, focused and important design problems than doing random innovation. The next sections provide concise details on the case study conducted on the AHS, and some of the results obtained on SBCE process implementation on rack subsystem.

\section{Case Study}

The steps have been conducted in one of the AHS designed by Carel Industries (which is called HumiFog, [13]). The system has been in the market for the last 10 years and primarily used for industrial applications (such as hospitals, residential buildings, textile factories, paint shops in car industries, and so on). It's priced at $8,700 €$ and sold all over the world. The main function of the system is to control the temperature and humidity levels. The product is the state of the art among other systems in terms of energy savings. The working principle is based on spraying atomized water mists at a high pressure (around 70 bars). The basic sub-systems are three: $C a b$ inet $(\mathrm{C})$, used to protect main components such as PLC (programmable logic control), water filters, water pump, etc.; Drop Separator (DS), used to achieve a highly purified water content and contains components such as module separators, frame support, housing, etc.; Rack (R), used to spray water to an ambient, and includes components as modulating and drain valves, nozzles for spraying, frames for support, and manifolds to carry nozzles and transport water. The SBCE IR steps for AHS are:

- Step 1: the requirements are classified in two levels, macro and micro, see Figure 2. Within each macro-requirement there are associated micro-requirements. Five macro-requirements are identified: (1) Technical performance $(\mathrm{P})$, which is related to the technical quality characteristics that the product should satisfy; (2) Usability (U), which is related to the product's simplicity during use; (3) Application (A), which is related to the flexibility of the system to be used in different applications (paint shop, data centers, hospitals, etc.); (4) Costs (C), aimed to reduce product and component costs; (5) Maintenance (M), to achieve easy repair of components and reduce the time between checkups. For each of the five macro-requirements, specific micro-requirements are defined. For P, U, A, C, and M, 11, 4, 5, 5, and 1 micro- requirements are identified respectively. For example, for usability (U): U1 (wide option range), wider operations during user-product interactions; U2 (fitting into customers sites); U3 (easy installation), reducing time to install at sites; U4 (friendly user interface). The designers conducted pairwise comparisons $(1,2 \ldots 9)$ within the categorized requirements, and AHP method is used to determine the weight vectors. The global weights (GWs) of micro-requirements are obtained by multiplying the weights of each with the weights of the corresponding macrorequirements (see Figure 2). 
- Step 2: four competitor products designed in Germany and Italy were evaluated for their performances. After the experts analyze the current HumiFog design with the competitors' designs for each customer requirement, they are assigned the degree of difficulties to fulfill the performance gaps identified (see Figure 2).

- Step 3/4: twenty three system contradictions are identified in the HumiFog system. Among which 22 are technical and 1 physical. For example, the contradiction T21 shown in Figure 2 is a physical contradiction between C5 (non-VDI requirement) with itself. The product has a European hygienic requirement called VDI 6022. However, once the company is expanding its business to different countries and sectors, VDI 6022 is not needed for some markets such as China or in some industrial applications (e.g. Tabaco industries). Thus, the contradiction type here is physical, where the product should stratify VDI 6022 requirement for some markets and applications, and in some others it is not necessary.
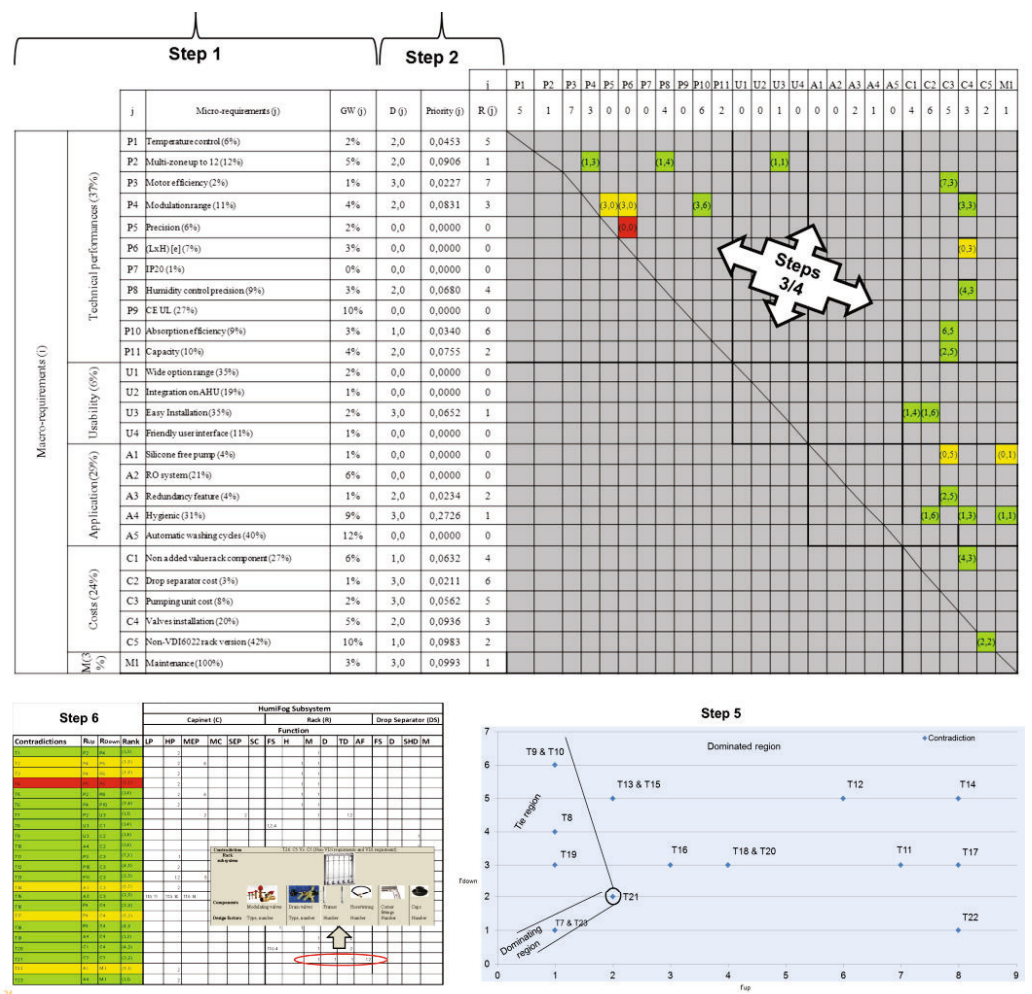

Fig. 2. Steps to build SBCE IR for HumiFog

- Step 5: based on the previous steps, ranking of independent contradictions can be made using the rules proposed in section 2. Looking at Figure 2, for example, T21 has dominated T13, 15, 16, 18, 20,12, 11, 14, 17, 22, is in tie with T19, 8, 9, 10 and is dominated by contradictions T7 and T23. On the other hand, root contradictions can be considered as priorities in case of selecting dependent ones. Thus, designers can make rationale choices to pursue SBCE processes to solve selected contradictions. 
- Step 6: once contradictions have been ranked and prioritized, designers can relate the selected contradictions with the associated physical design factors that need to be modified. For example, Figure 2 shows the design factors that have to be addressed to solve contradictions T21, the main subsystem that has to be modified is called rack, and its components design factors such as modulating valves (type and number), drain valves (type and number), the number of vertical frame needed, number of hoses needed, and number of corner fittings.

To experiment one SBCE process taking a contradiction, T21 is taken as a pilot. Although, it is dominated by other contradictions (T7 and T23) and it is in tie with others, T21 is considered simple by the designers taking into account the research time frame available during the preparation of this paper. In the next section, the result obtained in the SBCE application to solve contradiction T21 is presented.

\section{SBCE Process on Rack Subsystem}

As mentioned before, T21 is a physical contradiction where the VDI hygienic requirement is needed for some market and not for others. However, there is a growing market base that is requesting cheaper system without having the requirement, and thus the current configuration is overly designed. TRIZ principle of separation [4 and 6] is used to provide two different platforms for the two markets. Then, the SBCE process begins to remove non-value adding features for the non-VDI customers.

The target subsystem is rack which is used as a structure to distribute highly pressured water to an ambient. During operating condition, highly pressurized water will be pumped from pumping unit (motor and pump). The solenoid valves (normally open) modulate the water flow to vertical manifolds. Since the desired pressure level in the rack increases step by step, the water passes through many manifolds and the modulating valves are connected by hoses. Nozzles are used to spray the pressured water (mist) to the ambient. Holes which are covered with cups are provided along the manifolds for cleaning purposes. Once the rack subsystem finishes its operation, water comes out of the rack through drain valves.

- Concepts generation: three brainstorming workshops have been conducted to generate solutions to the non-VDI customers. Four concepts have been proposed: (1) frameless rack (2) changing the solenoid drain valves to mechanical valves, (3) using one solenoid valve for two manifolds, and (4) reducing the number of holes/ cups on the manifolds. In the first concept, the metal frames (used to slide the rack from the AHU) in the current design are removed and will be welded. Since the non-VDI clients will not require cleaning operations the concept is valid and $10 \%$ material cost reduction can be obtained. In the second concept, the VDI requirement strictly dictates the valves to be stainless steel (13€/piece), thanks to the separation of the markets, the drain valves used in the rack can be substituted with cheaper brass valves ( $5 € /$ piece), allowing $13 € /$ piece cost savings. Moreover, the current design embeds electrical control system (electrical wiring connected to valves) for precise water flow control, but the new design won't require the wiring 
systems, and thus cost reductions are possible. In the third concept, one modulating valve was dedicated to one manifold for higher hygienic quality water spray but the new design uses one valve for two manifolds. This concept allows number of valves to be reduced ( $20 €$ for every valve reduced). The last concept is to remove the holes and the cups used to cover the holes which have been added in the original design for frequent cleaning operations.

- Concepts testing: in SBCE process, testing concepts before detail design is paramount. It allows avoiding late design changes and helping to understand the limits of components to realize concepts. For example, mechanical brass valves from three suppliers were tested for concept 2. The nominal working pressure for drain valve is 70 bars, and any valve should withstand $10 \%$ more than the nominal. Moreover, the minimum pressure that any drain valve (which is normally open) will close should be investigated. If the minimum pressure that makes a valve to close is higher than 0.5 bars, there is the risk that the valve will not close when it is needed, and water kept draining. Once the components from three suppliers are tested, a supplier which passes both constraints will be chosen for bidding.

- Concepts merging: once concepts are generated and tested for constraints, the next step in SBCE process is convergence. In this particular case, the generated concepts can be merged to offer a high value rack platform for non-VDI customers. The new rack has $30 \%$ cost reduction compared to the original design which used to be overly designed for non-VDI clients. Thanks to the separation principle of TRIZ, it has been possible to eliminate non-value adding features from the current rack design. This new design has been taken as a project to be launched soon.

\section{$5 \quad$ Conclusion and Future Research}

The systematic methodology (SBCE IR) is proposed to identify and prioritize improvement areas where SBCE processes can be launched for matured products. It will help companies to avoid random approach to begin SBCE process and lead designers where innovation should take place to maximize customers' value. Moreover, the paper presents a case study on the AHS system (HumiFog) to validate the roadmap. Using it, the SBCE process on the rack subsystem to resolve contradiction T21 is discussed. The final result of $30 \%$ cost reduction is obtained by utilizing the SBCE IR, TRIZ principle of separation and followed by the SBCE process.

Some future researches can be identified. First, generating SBCE IR is time consuming; automating the steps will be next stage of the research. Second, the SBCE IR is based on evaluation of customer requirements, but in this type of market there are hierarchies of customers (contractors, installers, final customers) with different priorities for requirements. Finding ways to entertain in the best possible way the different customers in building the SBCE IR will be another possible research.

Acknowledgements. This work was partly funded by the European Commission through the Linked Design Project (FP7-2011-NMP-ICT-FoF, www . I inkeddesign . eu). The authors wish to acknowledge their gratitude and appreciation to the rest of the project partners for their contributions during the development of various ideas and concepts presented in this paper. 


\section{References}

1. Ward, A.C., Liker, J.K., Cristiano, J.J., Sobek, D.K.: The Second Toyota Paradox: How Delaying Decisions Can Make Better Cars Faster. Sloan Management Review 36, 43-61 (1995)

2. Sobek, D.K., Ward, A.C., Liker, J.K.: Toyota's Principles of Set-Based Concurrent Engineering. Sloan Management Review 40, 67-83 (1999)

3. Morgan, J.M., Liker, J.K.: The Toyota Product Development System: Integrating People, Process, and Technology. Productivity Press, New York (2006)

4. Altshuller, G.: Creativity as an Exact Science. Gordon \& Breach, New York (1984) ISBN 0-677-21230-5

5. Kowalick, J.: Psychological Inertia. TRIZ Journal (1998)

6. Altshuller, G.: The Innovation Algorithm: TRIZ, systematic innovation, and technical creativity. Technical Innovation Center, Worcester (1999) ISBN 0-9640740-4-4

7. McManus, H.L.: Product Development Value Stream Mapping (PDVSM) Manual. Lean Aerospace Initiative. Massachusetts Institute of Technology, Massachusetts (2005)

8. Rossi, M., Kerga, E., Taisch, M., Terzi, S.: Lean Product Development: Fact Finding Research in Italy. In: Proceedings of International Conference on Industrial Engineering and Systems Management (IESM), Metz, France, May 25-27 (2011)

9. Saaty, T.: The Analytic Hierarchy Process. McGraw-Hill (1980)

10. Yamashina, H., Ito, T., Kawada, H.: Innovative product development process by integrating QFD and TRIZ. Int. J. Prod. Res. 40(5), 1031-1050 (2002)

11. Mizuyama, H., Ishida, K.: Systematic decision making process for identifying the contradictions to be tracked by TRIZ to accomplish product innovation. Journal of Automation, Mobile Robotics \& Intelligent Systems 1(4) (2007)

12. Fontela, E., Gabus, A.: World Crisis - Decision Making Trial and Evaluation Laboratory. Futuribles (14), 211-221 (1978)

13. http://www.carel.com/carelcom/web/eng/catalogo/ prodotto_dett.jsp?id_gamma $=37 \& i d \_p r o d o t t o=377 \& i d \_m e r c a t o=2$ 